EPJ Web of Conferences 38, 17004 (2012)

DOI: $10.1051 /$ epjconf/20123817004

(C) Owned by the authors, published by EDP Sciences, 2012

\title{
Influence of proton shell closure on production of new superheavy nuclei
}

\author{
A.N. Kuzmina ${ }^{1}$, G.G. Adamian ${ }^{1,2}$, and N.V. Antonenko ${ }^{1}$ \\ ${ }^{1}$ Bogoliubov Laboratory of Theoretical Physics, Joint Institute for Nuclear Research, 141980 Dubna, Russia \\ ${ }^{2}$ Institute of Nuclear Physics, Tashkent, Uzbekistan
}

\begin{abstract}
The shell structure of nuclei with $Z>104$ is studied with modified two-center shell model. Using macroscopic-microscopic approach, the mass excesses and $Q_{\alpha}$-values are calculated and compared with available experimental data. Our calculations reveal quite strong shell effects at $Z=120-126$ as well as at $N=184$. If our predictions for the heaviest nuclei are correct then one can expect the production of evaporation residues with $Z \sim 120$ in the reactions ${ }^{50} \mathrm{Ti}+{ }^{249} \mathrm{Cf}$ and ${ }^{54} \mathrm{Cr}+{ }^{248} \mathrm{Cm}$ with the cross sections 23 and $10 \mathrm{fb}$, respectively. Nuclei with $Z=120$ and $N=178-182$ are expected to have $Q_{\alpha}$ about $11 \mathrm{MeV}$ and life-time of $0.17 \mathrm{~ms}-0.16 \mathrm{~s}$.
\end{abstract}

\section{Introduction}

To find ways of synthesis and identification of superheavy nuclei, the study of their structure is necessary $[1,2]$. The stability of superheavy nuclei is related to the shell effects which are ruled by the mean field and the spin-orbit interaction. Existing microscopic-macroscopic approaches [35] supply the basis for the intensive calculations of the properties of heavy nuclei.

The aim of this work is to apply the microscopic-macroscopic approach based on the two-center shell model TCSM [6] to the SHN and to reveal the trends in the shell effects, nuclear binding energies, $Q_{\alpha}$-values, quasiparticle states, and the evaporation residue cross sections with $Z$. The parameters of the TCSM were set so to describe the spins and parities of the ground state of known heavy nuclei (the rare earth nuclei, actinides, and some superheavy nuclei). This approach has been used in Ref. [7]. Note that the spectroscopic study of low-lying one- and two-quasiparticle states, and isomers in the heaviest nuclei [8-11] is up to date because of the problem of unambiguous identification of new superheavy nuclei $[1,12-14]$.

\section{Modified TCSM}

In our work, we use the shape parametrization adopted for the TCSM and use it for finding the single-particle levels in the ground state of the nucleus.

The contribution of an odd nucleon, occupying a singleparticle state $|\mu\rangle$ with energy $e_{\mu}$, to energy of a nucleus is described by the one-quasiparticle energy $\sqrt{\left(e_{\mu}-e_{F}\right)^{2}+\Delta^{2}}$. Here, the Fermi energy $e_{F}$ and the pairing-energy gap parameter $\Delta$ are calculated with the BCS approximation. Pairing interaction of the monopole type with strength parameters $G_{p}^{n}=\left(19.2 \mp 7.4 \frac{N-Z}{A}\right) A^{-1} \mathrm{MeV}$ [15] for neutrons and protons is used. For solving the pairing equations for $e_{F}$ and $\Delta$, the same prescription as in Ref. [15] is used. In the calculations of $e_{F}$ and $\Delta$ the blocking effect [16] is not taken explicitly into consideration. To take it effectively into account in the calculations of one-quasiparticle excitations $E_{\mu}=\sqrt{\left(e_{\mu}-e_{F}\right)^{2}+\Delta^{2}}-\sqrt{\left(e_{\mu}^{\prime}-e_{F}\right)^{2}+\Delta^{2}}$, where $e_{\mu}^{\prime}$ is the single-particle energy of occupied level below the Fermi level energies, we use the results of Ref. [16] where the reduction of $\Delta$ occurs by about factor of 0.85 . Calculating the potential energy surface as a function of collective coordinates with the TCSM, we find the ground-state potential minimum in which the energies of the low-lying one-quasiparticle states are obtained.

The details of the calculations of binding energies of nuclei in the ground states are presented in Ref. [5,7].

\section{Binding energies}

For the calculations of the binding energies of nuclei in the ground states we suggest the following expression:

$$
\begin{aligned}
B(Z, A) & =U\left(Z, A, \lambda_{g s}, \beta_{g s}\right)-a_{v}\left(1-a_{s}\left(\frac{N-Z}{A}\right)^{2}\right) A \\
& +W\left|\frac{N-Z}{A}\right|+\delta+c[(N-Z)-58],
\end{aligned}
$$

where $a_{s}=1.778$, the Wigner constant $W=30 \mathrm{MeV}$, $c=0.25 \mathrm{MeV}$ in the term correcting the charge-asymmetry energy, and the average pairing energy $\delta=4.8 / N^{1 / 3}+$ $4.8 / Z^{1 / 3}, 4.8 / N^{1 / 3}, 4.8 / Z^{1 / 3}$, and $0 \mathrm{MeV}$ for odd $Z$ and $N$, even $Z$ and odd $N$, odd $Z$ and even $N$, and even $Z$ and $N$, respectively.

\section{Calculations}

The calculated mass excesses $M_{t h}$, neutron separation energies $S_{n}$, shell corrections $E_{s h}$, and $Q_{\alpha}$-values you can see in [7] for nuclei with $105 \leq Z \leq 126$.

As seen in figure 1, the calculated $Q_{\alpha}$ are in a good, within $0.3 \mathrm{MeV}$, agreement with the available experimental $Q_{\alpha}^{\text {exp }}[1,2,12,17]$. The shell at $N=162$ is pronounced in our calculations as in Refs. [3,4]. The shell effects at $Z=$ 114 and $N=172-176$ provide rather weak dependence of $Q_{\alpha}$ on $N$. The strong role of the shell at $N=184$ is reflected in the well pronounced minimum of $Q_{\alpha}$. 

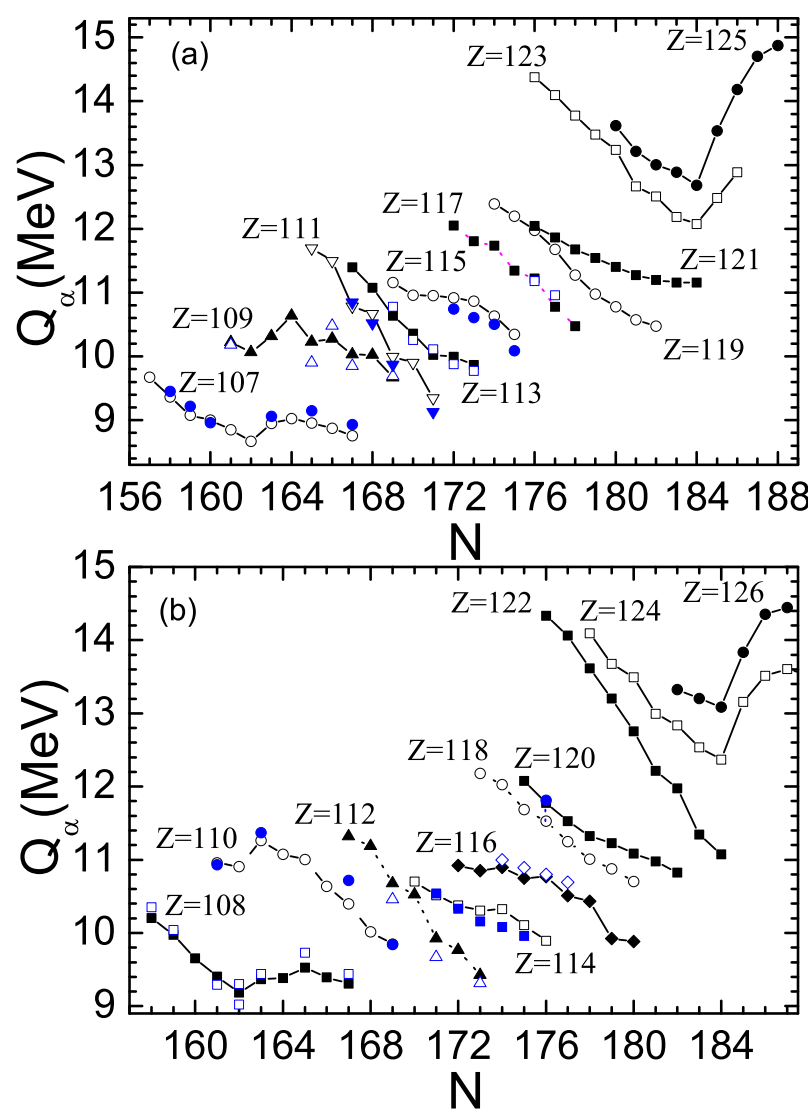

Fig. 1. (Color online) Calculated $\alpha$-decay energies (symbols connected by lines) are compared with available experimental data (symbols) $[1,2,12,17]$ for even- $Z$ (b) and odd- $Z$ (a) nuclei with $107 \leq Z \leq 126$.

The shell corrections prevent the fission of superheavy nuclei. The height $B_{f}$ of the fission barrier is mainly determined by the amplitude of the shell correction in the ground state for nuclei with $Z \geq 106$. As a result, $B_{f}$ strongly depends on the neutron and proton numbers of the compound nucleus, especially, on how close they are to the magic numbers.

The results of our calculation of $B_{f}-B_{n}$ as a function of $N$ are presented in figure 2 . As it is seen, our microscopicmacroscopic approach provides the shell at $Z=114$. However, the shell effects at $Z=120-126$ are rather strong. In figure 2, the fission barrier increases when $N$ approaches $N=184$. Since for nuclei with $Z=120-126$ the values of $Q_{\alpha}$ are minimal at $Z=120$ (figure 2) where the fission barriers are rather high (figure 2 ), the nuclei with $Z=120$ and $N=180-184$ are expected to be the most stable nuclei beyond those with $Z=114$ and $N=176-178$. The shell closure at $Z=120$ is expected in accordance with the relativistic mean-field model [18].

\section{Evaporation residue cross sections}

In the dinuclear system (DNS) model the dynamics of fusion is considered as a diffusion of the DNS in the mass asymmetry defined by $\eta=\left(A_{1}-A_{2}\right) /\left(A_{1}+A_{2}\right)\left(A_{1}\right.$ and $A_{2}$ are the mass numbers of the DNS nuclei) and in the internuclear distance $R$. The evaporation residue cross section
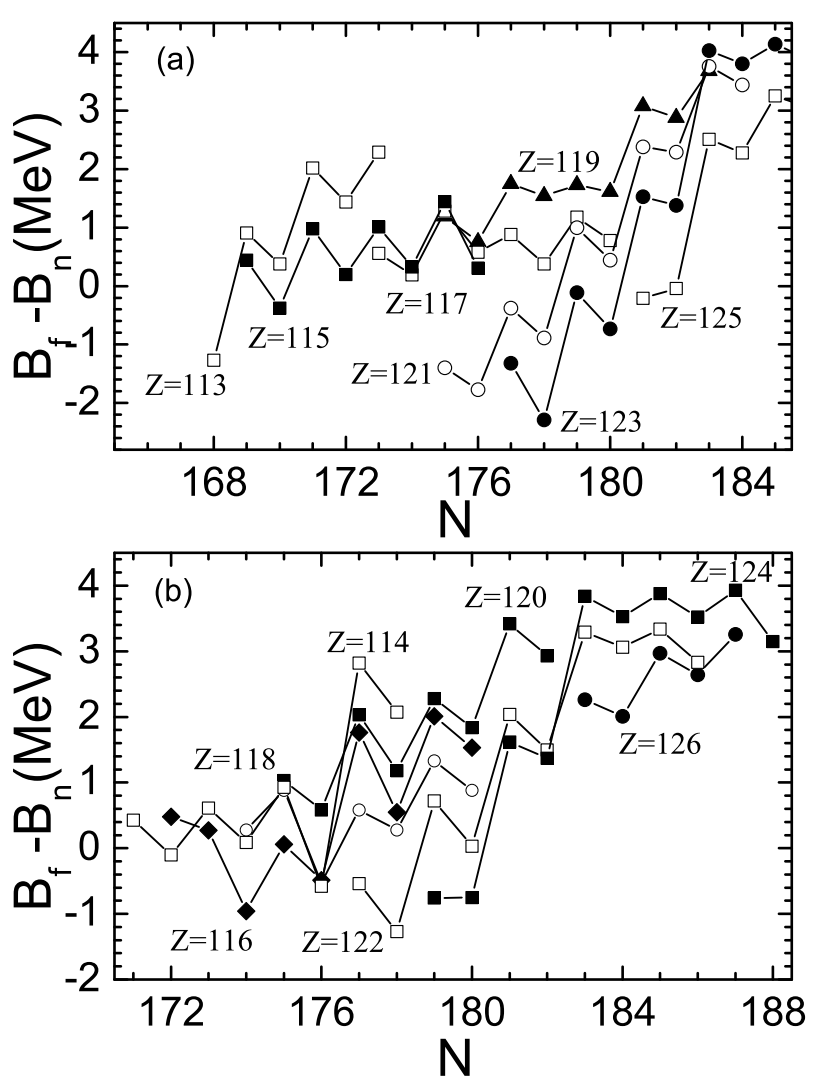

Fig. 2. The isotopic dependence of the value of $B_{f}-B_{n}$ with the data of [7]. The fission barrier $B_{f}$ is assumed to be an absolute value of the shell correction in the ground state of the nucleus. The results for the isotopes related to the indicated even- $Z$ (b) and odd- $Z$ (a) are shown by symbols connected by lines.

in $x n$ evaporation channel is determined as

$$
\begin{aligned}
\sigma_{E R}^{x n}\left(E_{\mathrm{c} . \mathrm{m} .}\right)= & \sum_{J} \sigma_{c}\left(E_{\mathrm{c} . \mathrm{m} .}, J\right) P_{C N}\left(E_{\mathrm{c} . \mathrm{m} .}, J\right) \\
& \times W_{s u r}^{x n}\left(E_{\mathrm{c} . \mathrm{m} .}, J\right)
\end{aligned}
$$

The capture cross section $\sigma_{c}\left(E_{\mathrm{c} . \mathrm{m} .}, J\right)$ defines the transition of the colliding nuclei through the Coulomb barrier and the formation of the DNS when the kinetic energy above the barrier is transformed into the excitation energy of the DNS and the angular momentum $J$ of the relative motion is redistributed in the DNS. For the reactions treated, the maximum evaporation residue cross sections are at $E_{\mathrm{c} . \mathrm{m} .}$ at which the collisions for all orientations of deformed nuclei become possible. Therefore, in our case there is no noticeable loose of the cross section during the capture.

Using our predictions of nuclear properties in [7], we calculated the evaporation residue cross sections in the reactions ${ }^{48} \mathrm{Ca},{ }^{50} \mathrm{Ti},{ }^{54} \mathrm{Cr},{ }^{58} \mathrm{Fe},{ }^{64} \mathrm{Ni}+{ }^{238} \mathrm{U},{ }^{244} \mathrm{Pu},{ }^{248} \mathrm{Cm}$, ${ }^{249} \mathrm{Cf}$ (figure 3 ). In comparison to our previous calculations with the mass table of Ref. [3], in figure 3 the values of $\sigma_{E R}$ decreases slower with increasing $Z$. The stronger shell effect revealed here for nuclei with $Z>118$ result larger survival probabilities and larger values of $\sigma_{E R}$. In the reactions ${ }^{48} \mathrm{Ca}+{ }^{238} \mathrm{U},{ }^{248} \mathrm{Cm},{ }^{249} \mathrm{Cf}$ the experimental values of $\sigma_{E R}^{3 n}$ are $0.5-2.5 \mathrm{pb}$, about $1 \mathrm{pb}$, and $0.5 \mathrm{pb}$ [1], respectively. Thus, the difference between the calculated and experimental $\sigma_{E R}$ are within the experimental and theoretical uncertainties. A good description of existing data allows us 


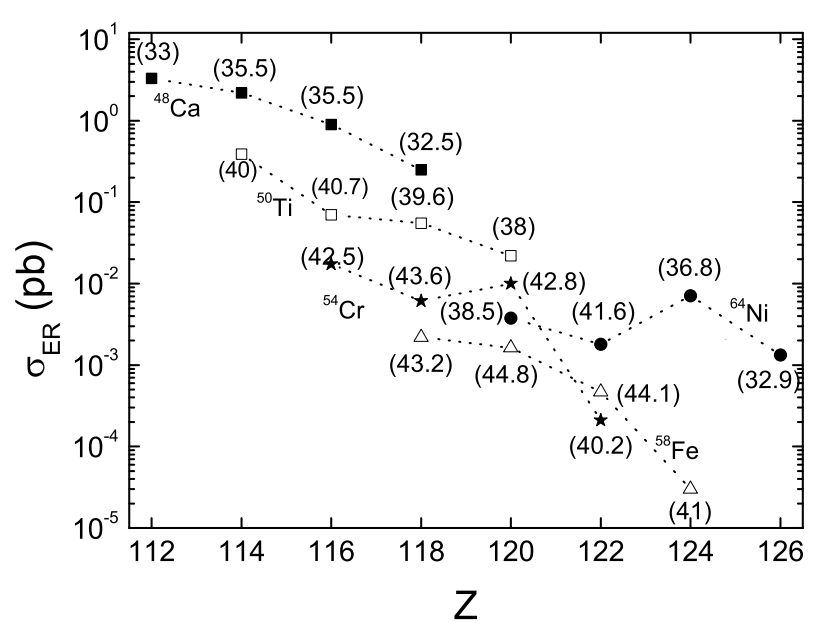

Fig. 3. The evaporation residue cross sections in the maxima of excitation functions versus charge number $Z$ for the reactions ${ }^{48} \mathrm{Ca},{ }^{50} \mathrm{Ti},{ }^{54} \mathrm{Cr},{ }^{58} \mathrm{Fe},{ }^{64} \mathrm{Ni}+{ }^{238} \mathrm{U},{ }^{244} \mathrm{Pu},{ }^{248} \mathrm{Cm},{ }^{249} \mathrm{Cf}$. The predicted properties of superheavy nuclei from [7] are used. The excitation energies of compound nuclei are given in brackets.

to be reliable in the predictions for the reactions with projectiles heavier than ${ }^{48} \mathrm{Ca}$.

With ${ }^{50} \mathrm{Ti}$ beam the values of $\sigma_{E R}$ for the nuclei with $Z=114-118$ are expected to be 5-10 times smaller than those resulted by ${ }^{48} \mathrm{Ca}$ beam. The main reason for this is the decrease of $P_{C N}$ in Eq.(2) with mass asymmetry in the entrance channel of reaction. With ${ }^{50} \mathrm{Ti}$ the nucleus ${ }^{295} 120$ is predicted to be produced with the cross section of $23 \mathrm{fb}$. In the ${ }^{54} \mathrm{Cr}+{ }^{248} \mathrm{Cm}$ reaction the compound nucleus would have 3 neutrons more than in the ${ }^{50} \mathrm{Ti}+{ }^{249} \mathrm{Cf}$ reaction. Therefore, the decrease of $P_{C N}$ is partly negated by the increase of $W_{\text {sur }}$ and the nucleus ${ }^{298} 120$ can be produced with the cross section of $10 \mathrm{fb}$. For the production of nuclei with $Z=122-126,{ }^{64} \mathrm{Ni}$ beam would lead to larger cross sections, $1-8 \mathrm{fb}$.

Using our predictions of nuclear properties, we calculated the evaporation residue cross sections of the reactions ${ }^{50} \mathrm{Ti}+{ }^{A} \mathrm{Cf}$ and ${ }^{54} \mathrm{Cr}+{ }^{A} \mathrm{Cm}$ figures 4 and 5. At zero excitation energy, the predicted values of fission barriers used in the calculations are in the energy interval $8.1-10.1 \mathrm{MeV}$. Note that for the reactions ${ }^{48} \mathrm{Ca}+{ }^{238} \mathrm{U},{ }^{244} \mathrm{Pu},{ }^{248} \mathrm{Cm},{ }^{249} \mathrm{Cf}$ the calculated and experimental values of evaporation residue cross sections are quite close [19]. A good description of existing data allows us to be confident in the predictions for the reactions with heavier projectiles. In the ${ }^{50} \mathrm{Ti}+{ }^{249} \mathrm{Cf}$ $(Q=-194.75 \mathrm{MeV})$ reaction the nucleus ${ }^{295} 120$ is predicted to be produced in a $3 n$ evaporation channel with a cross section of $23 \mathrm{fb}$. In the ${ }^{54} \mathrm{Cr}+{ }^{248} \mathrm{Cm}(Q=-205.59 \mathrm{MeV}) \mathrm{re}-$ action the compound nucleus would have 3 neutrons more than in the ${ }^{50} \mathrm{Ti}+{ }^{249} \mathrm{Cf}$ reaction. Therefore, the decrease of $P_{C N}$ is partly negated by the increase of $W_{\text {sur }}$, and the nucleus ${ }^{298} 120$ is predicted to be produced with a cross section of $10 \mathrm{fb}$ ( $4 n$ evaporation channel). As in Refs. [2022], the isotopic dependence of $\sigma_{E R}$ is rather weak in the treated interval of mass numbers $A$. Indeed, the values of $\sigma_{E R}$ are almost the same in the cases of ${ }^{246} \mathrm{Cm}(Q=-208.07$ $\mathrm{MeV}$ ) and ${ }^{248} \mathrm{Cm}$ as target. There is a certain interval of mass numbers of target nuclei where the product $P_{C N} W_{\text {sur }}$ changes only weakly [20-22].

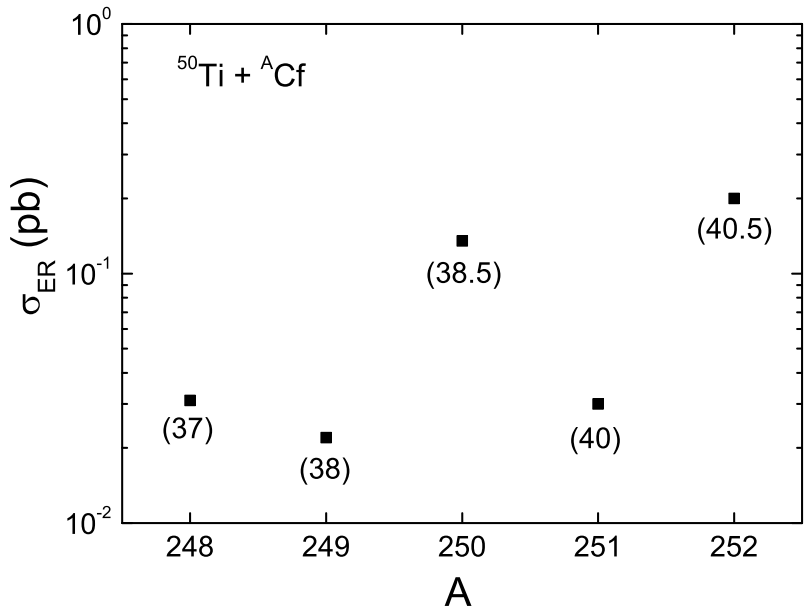

Fig. 4. Evaporation residue cross sections of the maxima of excitation functions of the reactions ${ }^{50} \mathrm{Ti}+{ }^{A} \mathrm{Cf}$ versus $A$. The excitation energies of compound nuclei are given in brackets. Groundstate mass excesses $M_{\mathrm{th}}=211.8,213.05,213.76,215.15$, and $216.05 \mathrm{MeV}$ for the nuclei ${ }^{298} 120,{ }^{299} 120,{ }^{300} 120,{ }^{301} 120$, and ${ }^{302} 120$, respectively, were used in the calculations.

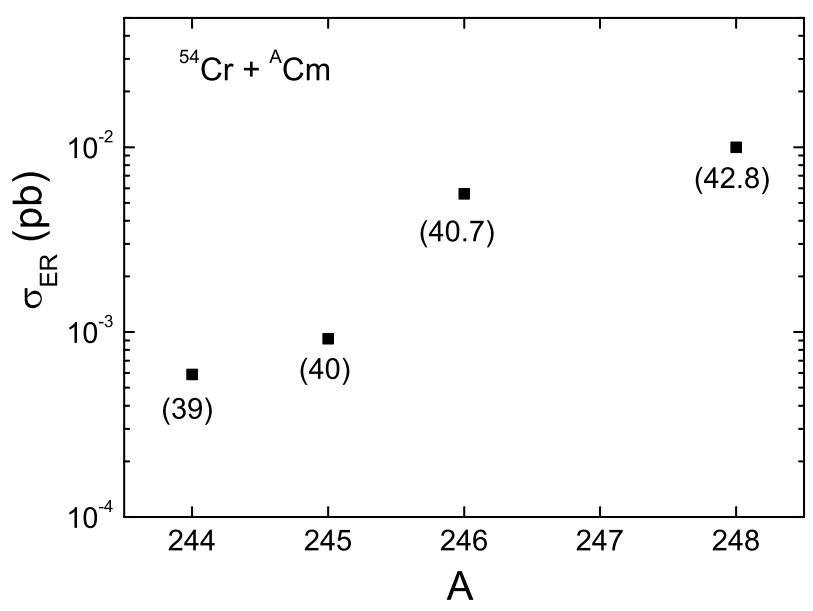

Fig. 5. The same as in figure 4, but for the reactions ${ }^{54} \mathrm{Cr}+{ }^{A} \mathrm{Cm}$.

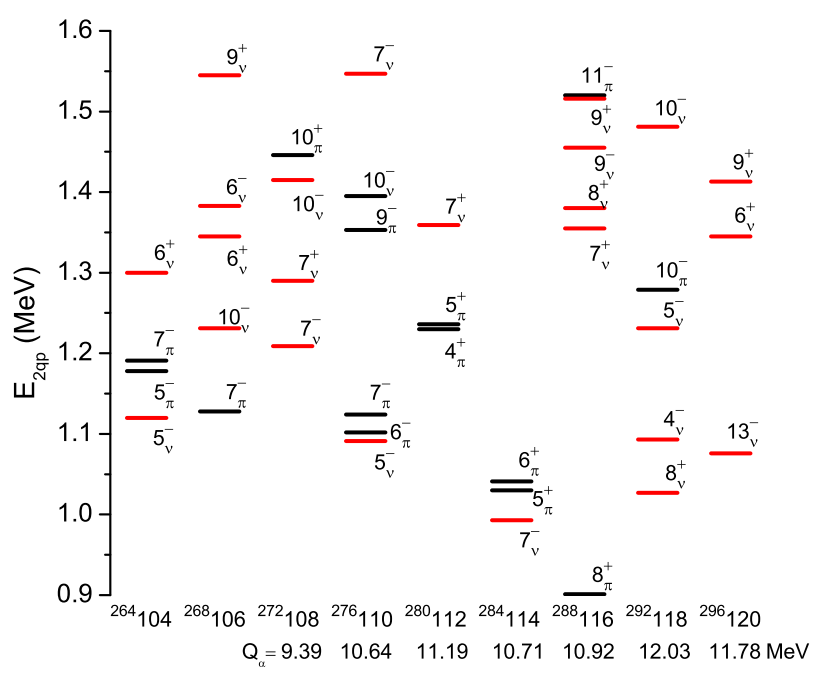

Fig. 6. Calculated energies of low-lying two-quasiproton ( black signs) and two-quasineutron (red signs) states in the indicated nuclei of the $\alpha$-decay chain of ${ }^{296} 120$. The resulting $K$ values are indicated. 
EPJ Web of Conferences

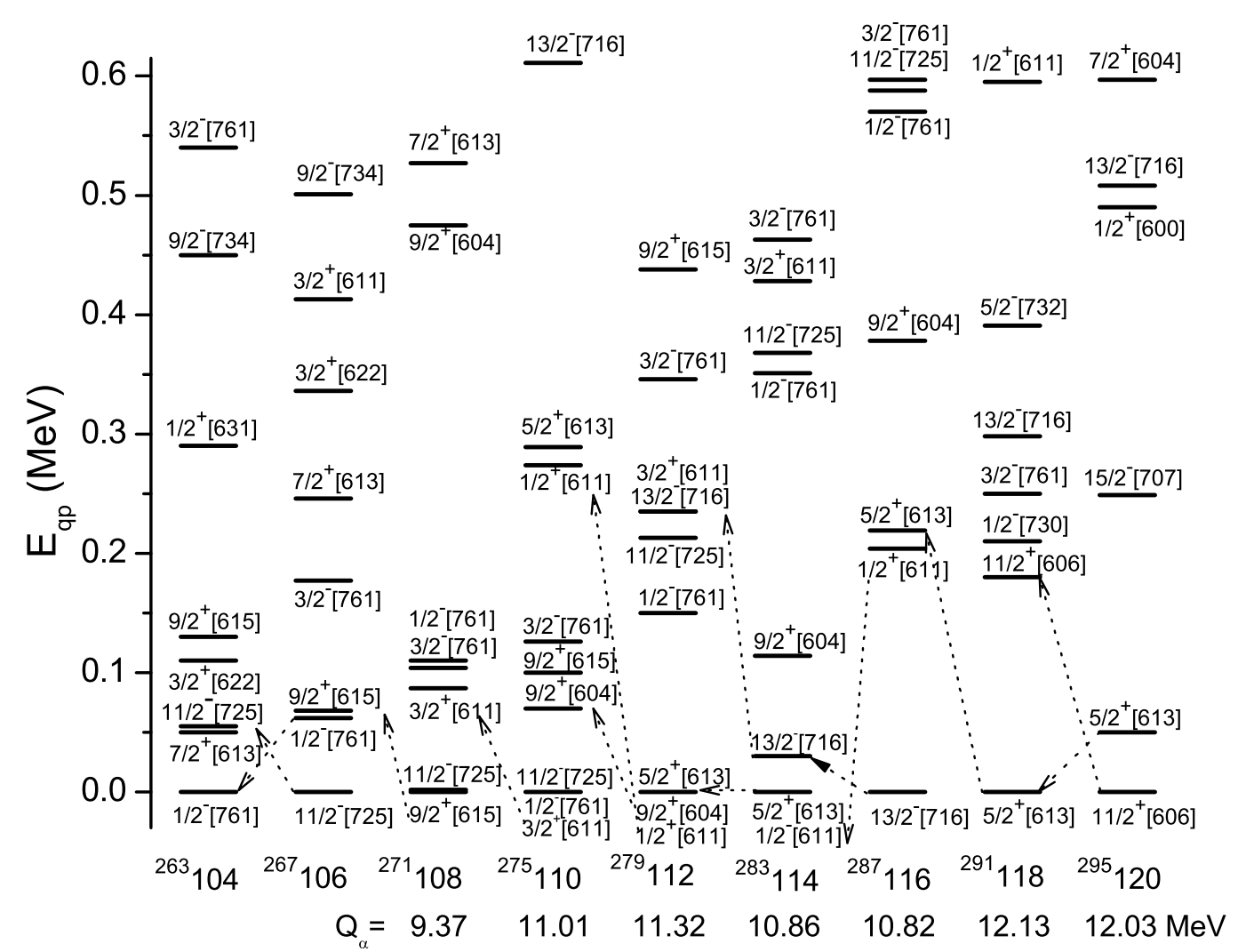

Fig. 7. Calculated energies of low-lying one-quasineutron states in the indicated nuclei of the $\alpha$-decay chain of ${ }^{295} 120$. The states are marked by the Nilsson asymptotic quantum numbers. The possible $\alpha$ decays are shown by arrows.

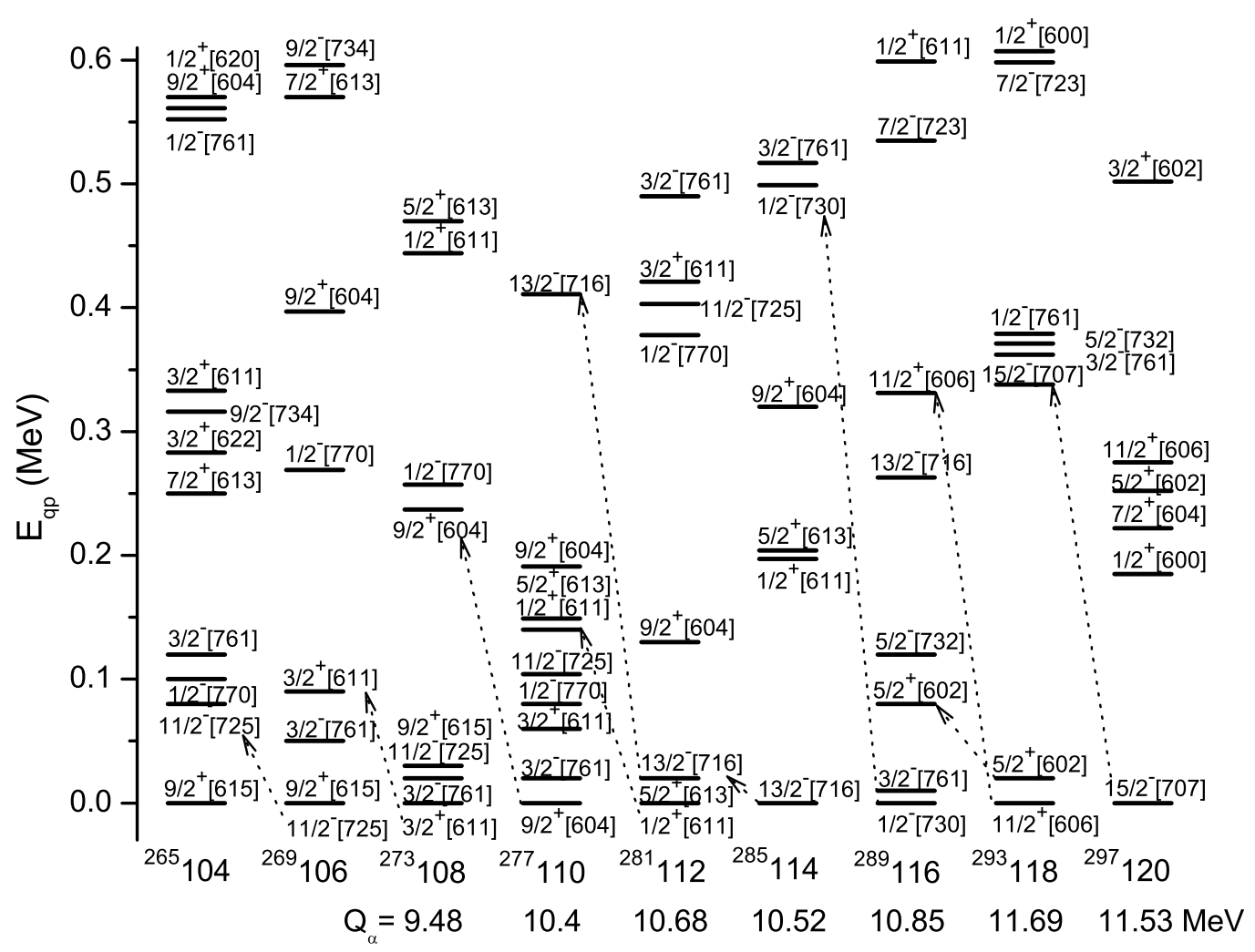

Fig. 8. The same as in figure 7, but for the nuclei of the $\alpha$-decay chain of ${ }^{297} 120$. 


\section{One- and two-quasiparticle states}

In Ref. [23] we treated the isotopes of superheavy nuclei with even $Z$ which can be reached in complete fusion reactions with available stable projectiles and targets. The results for odd $Z$ nuclei are given in Ref. [19].

In figure 6 the energies of two-quasiparticle states are presented for the nuclei of $\alpha$-decay chain of ${ }^{296} 120$. While for nuclei with $Z \leq 118$ the first two-quasiproton states have energies smaller than $1.2 \mathrm{MeV}$, in ${ }^{296} 120$ the energies of the first two-quasiproton states are at about $1.9 \mathrm{MeV}$. This indicates a larger gap in the proton single-particle spectrum. So, the shell effects become stronger beyond $Z=114$. The $\alpha$-decay chain starting from ${ }^{296} 120$ probably terminates at ${ }^{284} 114$ by spontaneous fission. Indeed, ${ }^{284} 114$ is predicted to decay by spontaneous fission in $0.012 \mathrm{~s}$ [24] whereas $\alpha$ decay is expected to have a longer half-life of $0.045 \mathrm{~s}$.

The lowest two-quasiparticle isomeric states in ${ }^{296} 120$, ${ }^{292} 118,{ }^{288} 116$, and ${ }^{284} 114$ are $13_{v}^{-}\left\{11 / 2^{+}[606] \otimes 15 / 2^{-}[70\right.$ $7]\}, 8_{v}^{+}\left\{5 / 2^{+}[602] \otimes 11 / 2^{+}[607]\right\}, 8_{\pi}^{-}\left\{7 / 2^{-}[503] \otimes 9 / 2^{-}[50\right.$ $5]\}$, and $7_{v}^{-}\left\{1 / 2^{+}[611] \otimes 13 / 2^{-}[716]\right\}$, respectively (figure 6).

The $\alpha$-decay chains of 295,297120 are expected to be long, at least up to Rf, because the spontaneous fission of odd nuclei is hindered and $\alpha$ decays occur faster [1]. In figures 7,8 we show the calculated one-quasiparticle spectra of nuclei of $\alpha$-decay chains of ${ }^{295,297} 120$. The possible $\alpha$ decays are marked. As seen in figure 7, the $\alpha$ decay of ${ }^{291} 116$ is hindered, because the corresponding levels have high energies in the daughter nucleus.

Since in figures 7,8 the nuclei with $Z>108$ are only slightly deformed, the quasiparticle spectra are rather dense near the ground states and the appearance of isomeric states is likely. Alpha decays can occur from these isomeric states if they live longer with respect to $\gamma$ decay. The $\alpha$ decays from the isomeric states of ${ }^{295,297} 120$ are calculated to be faster than from the ground states.

\section{Summary}

The calculations performed with the modified TCSM reveal quite strong shell effects at $Z=120-126$. The strong shell effect is at $N=184$. So, our macroscopic- microscopic treatment qualitatively leads to the results close to those in the mean-field treatments. The shell structure of heaviest nuclei predicted with macroscopic-microscopic model crucially depends on the parameters of single-particle potential and spin-orbit interaction. If our predictions of the structure of heaviest nuclei are correct, than one can expect the production of evaporation residues with $Z=120$ in the reactions ${ }^{50} \mathrm{Ti}+{ }^{249} \mathrm{Cf}$ and ${ }^{54} \mathrm{Cr}+{ }^{248} \mathrm{Cm}$ with the cross sections 23 and $10 \mathrm{fb}$, respectively. The $Z=120$ nuclei with $N=178-182$ are expected to have $Q_{\alpha}$ about 11 $\mathrm{MeV}$ and life time of about $1.7 \mathrm{~ms}-0.16 \mathrm{~s}$. Note that the definition of maxima of the excitation functions provide the good test for the predictions of the models.

This work was partly supported by the DFG and RFBR.

\section{References}

1. Yu.Ts. Oganessian, J. Phys. G: Nucl. Part. Phys. 34, R165 (2007)
2. Yu.Ts. Oganessian, et al., Phys. Rev. Lett. 104, 142502 (2010)

3. P. Möller, J.R. Nix, W.D. Myers, and W.J. Swiatecki, At. Data Nucl. Data Tables 59, 185 (1995)

4. I. Muntian, Z. Patyk, and A. Sobiczewski, Acta. Phys. Pol. B 32, 691 (2001); 34, 2141 (2003); I. Muntian, S. Hofmann, Z. Patyk, and A. Sobiczewski, Acta. Phys. Pol. B 34, 2073 (2003); Phys. At. Nucl. 66, 1015 (2003); A. Parkhomenko, I. Muntian, Z. Patyk, and A. Sobiczewski, Acta. Phys. Pol. B 34, 2153 (2003); A. Parkhomenko and A. Sobiczewski, Acta. Phys. Pol. B 36, 3115 (2005)

5. G.G. Adamian, N.V. Antonenko, and W. Scheid, Phys. Rev. C 81, 024320 (2010); Acta Phys. Pol. B 40, 759 (2009); AIP Conf. Proc. 1165, 136 (2009); G.G. Adamian, N.V. Antonenko, S.N. Kuklin, and W. Scheid, Phys. Rev. C 82, 054304 (2010); G.G. Adamian, N.V. Antonenko, S.N. Kuklin, B.N. Lu, L.A. Malov, and S.G. Zhou, Phys. Rev. C 84, 024324 (2011)

6. J. Maruhn and W. Greiner, Z. Phys. A 251, 431 (1972)

7. A.N. Kuzmina, G.G. Adamian, N.V. Antonenko, and W. Scheid, Phys. Rev. C 85, 014319 (2012)

8. R.-D. Herzberg and P.T. Greenlees, Prog. Part. Nucl. Phys. 61, 674 (2008); R.-D. Herzberg and D.M. Cox, Radioachim. Acta 99, 441 (2011)

9. F. P. Hessberger, Eur. Phys. J. D 45, 33 (2007); F.P. Hessberger, et al., Eur. Phys. J. A 41, 145 (2009); F.P. Hessberger, et al., Eur. Phys. J. A 43, 175 (2010)

10. S. Antalic, et al., Eur. Phys. J. A 43, 35 (2010); B. Streicher, et al., Eur. Phys. J. A 45, 275 (2010); M.Venhart, et al., Eur. Phys. J. A 47, 20 (2011); S. Antalic, et al., Eur. Phys. J. A 47, 62 (2011)

11. S.K. Tandel, et al., Phys. Rev. C 82, 041301 (2010); A. Lopez-Martens et al., Nucl. Phys. A852, 15 (2011)

12. S. Hofmann, et al., Eur. Phys. J. A 32, 251 (2007); S. Hofmann, Lec. Notes Phys. 764, 203 (2009); Radiochim. Acta 99, 405 (2011)

13. S. Heinz, et al., J. Phys.: Conf. Ser. 282, 012007 (2011); S. Hofmann et al., Eur. Phys. J. A 48, 62, (2012)

14. S. Hofmann and G. Münzenberg, Rev. Mod. Phys. 72, 737 (2000)

15. S. G. Nilsson and I. Ragnarsson, Shapes and Shells in Nuclear Structure (Cambridge University Press, Cambridge 1995)

16. V. G. Soloviev, Theory of Complex Nuclei (Pergamon Press, Oxford, 1976)

17. L. Stavsetra, K.E. Gregorich, J. Dvorak, P.A. Ellison, I. Dragojević, M.A. Garcia, and H. Nitsche, Phys. Rev. Lett. 103, 132502 (2009)

18. P.G. Reinhard, Rep. Prog. Phys. 52, 439 (1989); P. Ring, Prog. Part. Nucl. Phys. 37, 193 (1996); M. Bender, P.H. Heenen, and P.G. Reinhard, Rev. Mod. Phys. 75, 121 (2003)

19. A.N. Kuzmina, G.G. Adamian, and N.V. Antonenko, Phys. Rev. C 85, 017302 (2012)

20. G.G. Adamian, N.V. Antonenko, and W. Scheid, Phys. Rev. C 69, 011601(R) (2004)

21. G.G. Adamian, N.V. Antonenko, and W. Scheid, Phys. Rev. C 69, 014607 (2004)

22. G.G. Adamian, N.V. Antonenko, and W. Scheid, Phys. Rev. C 69, 044601 (2004) 


\section{EPJ Web of Conferences}

23. A.N. Kuzmina, G.G. Adamian, and N.V. Antonenko, Eur. Phys. J. A 47, 145 (2011)

24. R. Smolańczuk, J. Skalski, and A. Sobiczewski, Phys. Rev. C 52, 1871 (1995) 